\title{
NONRECIPROCAL EFFECT IN MAGNETOOPTICAL-NONLINEAR WAVEGUIDE
}

\author{
K. GNIADEK \\ Faculty of Physics, Warsaw University of Technology \\ Koszykowa 75, 00-662 Warsaw, Poland
}

\begin{abstract}
The nonreciprocal phase shift of TM waves guided by the linear thin film sandwiched between magnetooptic semi-infinite substrate and nonlinear Kerr-type semi-infinite cladding has been investigated theoretically and numerically. With the help of the conservation energy law for TM modes in nonlinear waveguide the dispersion relation without a knowledge of the nonlinear field profile has been derived. The dependence of the nonreciprocal behaviour of the guided waves in terms of the local intensity at the film-nonlinear cladding boundary is discussed. Introducing a nonlinear material into magnetooptic waveguide structure leads to interesting applications for a new class of integrated devices which combine magnetooptic and nonlinear effects.
\end{abstract}

PACS numbers: 42.65.Wi

\section{Introduction}

The magnetooptical waveguides have been receiving a great deal of attention because they have the application as basic elements for the nonreciprocal devices realized in a planar form [1-3]. The nonreciprocal devices such as isolators and circulators are needed to stabilize the semiconductor-laser operation to avoid self-oscillation of the laser due to reflection in the communication system. Most proposals for waveguide-type isolators are based on nonreciprocal TE-TM mode conversion [4]. In order to achieve the efficient TE-TM conversion, the precise phase matching between the interacting modes is necessary by orienting the optic axis of the materials precisely and by controlling the thickness of the film accurately. These requirements are difficult to fulfil in practice. The other concept for an optical isolator is based on the nonreciprocal phase shift for TM modes in magnetooptical waveguide in the so-called Voigt (or equatorial) configuration [5-7]. It was shown that a propagation constant of the TM mode is different for the propagation in the opposite direction in the presence of dc magnetic field applied perpendicular to the direction of the propagation. This concept has the advantages 
because it involves only one polarization namely TM and therefore is no need to consider phase matching.

The purpose of this paper is to present a new configuration of the magnetooptic layered structure in which one medium (e.g. top layer) is nonlinear. Nonlinear optical waveguides exhibit a number of interesting properties that have been studied extensively in the recent years (see, for example [7] and references in review articles [8-10]) because of their potential applications in all-optical signal processing. To date, closed form solutions have been obtained only for TE-polarized waves. For the TM waves, the presence of two electric field components, one parallel and one perpendicular to the interfaces, makes the analysis considerably complicated. To obtain an analytical description of the TM-case a number of approximations have been employed $[11,12]$.

Inserting the nonlinear material into magnetooptic waveguide gives an additional "degree of freedom" namely a power flow down the guide $[13,14]$. The power dependence of the propagation constant allows a good control of the nonreciprocal behaviour of the counterpropagating TM modes. This shows a great potential for application of the magnetooptic-nonlinear waveguide in the design and the realization of a new class of integrated devices utilizing both magnetooptic and nonlinear effects. As an example such device can be a nonreciprocal limiter of the guided power flow. Another possible device is the intensity-controlled integrated isolator based on the magnetooptic directional coupler [15]. In such structure, the nonlinearity acts on the propagation constants of the counterpropagating TM modes modifying the coupling condition, and therefore the transfer efficiency which can be optimized, say in the backward direction and reduced for the forward propagation.

\section{Dispersion relation for TM modes}

The guiding structure to be considered consists of three layers - the magnetooptic substrate, the linear nonmagnetic film, and the nonlinear top layer. The external dc magnetic field $H_{0}$ is applied parallel to the film plane and perpendicular to the direction of propagation which is along the $z$-axis (the so-called Voigt configuration). Neglecting losses, the corresponding dielectric tensor of magnetooptic (gyrotropic) material has the following form:

$$
\varepsilon^{\mathrm{MO}}=\left(\begin{array}{ccc}
\varepsilon_{x} & 0 & \mathrm{i} \delta \\
0 & \varepsilon_{y} & 0 \\
-\mathrm{i} \delta & 0 & \varepsilon_{z}
\end{array}\right) .
$$

The nondiagonal elements $\delta$ are related to the specific Faraday rotation $\theta_{\mathrm{F}}$ by $\delta=\theta_{\mathrm{F}} \sqrt{\varepsilon_{z}} \lambda / \pi[6]$. Gyrotropy is induced by an applied magnetic field.

It is well known that the modes in the Voigt configuration can be separated into $\mathrm{TE}\left[E_{y}, H_{z}, H_{z}\right]$ and $\mathrm{TM}\left[H_{y}, E_{x}, E_{z}\right]$ polarizations. The TE and TM modes are not coupled. Properties of the TE modes do not depend on the sign of the propagation direction and are preserved in the presence of the externally applied magnetic field. We consider only TM modes as TM modes are nonreciprocal in the Voigt configuration. For simplicity, the magnetooptic medium is assumed to 
be isotropic $\varepsilon_{x}=\varepsilon_{y}=\varepsilon_{z}=\varepsilon_{s}$. We suppose that the nonlinear cladding is of the Kerr-type and isotropic, so that the nonlinear dielectric subtensor for the TM waves reads as

$$
\varepsilon^{\mathrm{NL}}=\left(\begin{array}{cc}
\varepsilon_{x x} & 0 \\
0 & \varepsilon_{z z}
\end{array}\right),
$$

where the elements $\varepsilon_{x x}$ and $\varepsilon_{z z}$ depend on the local intensity and we assume

$$
\varepsilon_{x x}=\varepsilon_{z z}=\varepsilon^{\mathrm{NL}}=\varepsilon_{\mathrm{L}}+\alpha\left(\left|E_{x}\right|^{2}+\left|E_{z}\right|^{2}\right)
$$

(electrostrictive or thermal nonlinearity) where $\varepsilon_{\mathrm{L}}$ is a linear part of the dielectric permittivity and $\alpha$ is the nonlinear coefficient.

The electric and magnetic field vectors for TM waves propagating along the $z$-axis with the angular frequency $\omega$ and propagation constant $\beta$ are written as

$$
\begin{aligned}
& \boldsymbol{E}(x, z)=\left[E_{x}(x) \boldsymbol{i}_{x}+E_{z}(x) \boldsymbol{i}_{z}\right] \exp [\mathrm{i}(\omega t-\beta z)], \\
& \boldsymbol{H}(x, z)=H_{y}(x) \boldsymbol{i}_{y} \exp [\mathrm{i}(\omega t-\beta z)] .
\end{aligned}
$$

For the magnetooptic medium $(x<0)$ the magnetic field amplitude $H_{y}(x) \equiv H(x)$ is a solution of the wave equation

$$
\frac{\mathrm{d}^{2} H}{\mathrm{~d} x^{2}}+\left(k_{0}^{2} n_{\mathrm{MO}}^{2}-\beta^{2}\right) H=0,
$$

where $n_{\mathrm{MO}}=\sqrt{\left(\varepsilon_{s}^{2}-\delta^{2}\right) / \varepsilon_{s}}$ is the effective index of refraction of magnetooptic medium and $k_{0}$ is the free-space wave number. In the nonlinear cladding $(x>d)$ the field amplitude $H(x)$ obeys the equation

$$
\frac{d}{\mathrm{~d} x}\left(\frac{1}{\varepsilon^{\mathrm{NL}}} \frac{\mathrm{d} H}{\mathrm{~d} x}\right)+\left(k_{0}^{2} \varepsilon^{\mathrm{NL}}-\beta^{2}\right) \frac{H}{\varepsilon^{\mathrm{NL}}}=0 .
$$

The second order nonlinear differential equation (1.6) can be solved in quadratures for an arbitrary form of the local intensity-dependent dielectric function $\varepsilon^{\mathrm{NL}}$. The corresponding electric field $\boldsymbol{E}$ can be calculated from the Maxwell equations. Assuming the decaying field in the magnetooptic substrate and taking into account the conservation law in the nonlinear medium

$$
\left|E_{x}\right|^{2}\left[-2\left(k_{0} / \beta\right)^{2} \eta^{2}+3 \eta-1\right]-\left|E_{z}\right|^{2}(\eta+1)=\mathrm{const}
$$

[14] and applying the boundary condition at the film-substrate and film-cover interfaces one obtains the relation

$$
\begin{array}{r}
k_{0} d \sqrt{n_{f}^{2}-N^{2}}=\arctan \left(\frac{n_{f}}{n_{s}}\right)^{2} \frac{\sqrt{N^{2}-n_{s}^{2}}-N \delta / \varepsilon_{s}}{\sqrt{n_{f}^{2}-N^{2}}} \\
+\arctan \left(\frac{\varepsilon_{f}}{\varepsilon_{c} \eta} \sqrt{\left.\frac{(3 \eta-1)\left(N^{2}-\eta^{(\mathrm{NL})^{2}}\right)}{(\eta+1)\left(n_{f}^{2}-N^{2}\right)}\right)+m \pi}\right.
\end{array}
$$

where $d$ is the film thickness, $N=\beta / k_{0}$ is the effective index of refraction (the mode index) and

$$
n_{f}^{2}=\varepsilon_{f}, \quad n_{c}^{2}=\varepsilon_{\mathrm{L}}, \quad \eta^{(\mathrm{NL}) 2}=\frac{2 n_{c}^{2} \eta^{2}}{3 \eta-1}
$$


$\eta=\varepsilon^{\mathrm{NL}} / \varepsilon_{\mathrm{L}}=1+\left(\alpha / \varepsilon_{c}\right)\left(\left|E_{x c}\right|^{2}+\left|E_{z e}\right|^{2}\right)$ is the normalized boundary value of the permittivity of nonlinear medium.

Equation (1.8) is the dispersion relation of TM modes which determines the propagation constant $\beta$. The nonreciprocity of TM modes is evident from Eq. (1.8). Due to the off-diagonal elements $\delta$ in the dielectric tensor (1.1), the term linear in $\beta$ enters this equation. Thus, the absolute value of the propagation constant is different for the propagation in the opposite directions, depending on the sign of $\beta$. We note that changing the $\delta$ sign (reversing the direction of dc magnetic field) has the same effect as changing the sign of $\beta$. It follows also from the dispersion relation that the propagation constants depend on the guided power flow (via the power density at the film-cover interface). Note that the limit $\eta \rightarrow 1$ in Eq. (1.8) yields the dispersion relation for linear magnetooptic waveguide [5].

\section{Numerical results}

First, we demonstrate the nonreciprocal behaviour of TM modes in a linear magnetooptic waveguide. The numerical results were calculated for the following parameters: $\varepsilon_{s}=5.2, \varepsilon_{f}=5.5, \varepsilon_{\mathrm{L}}=4.0, \delta=0.05$. To accentuate the effect of the nonreciprocity the value for $\delta$ is chosen two orders of magnitude larger than a typical value of $\delta$.

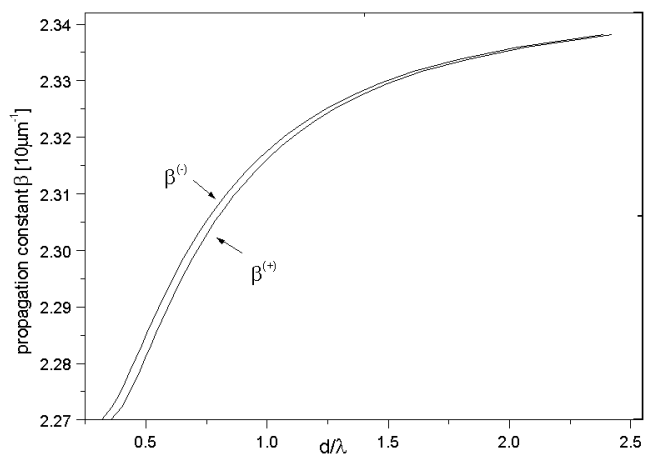

Fig. 1. Propagation constants of the forward $\beta^{(+)}$and backward $\beta^{(-)}$TMo modes in the magnetooptic waveguide versus the film thickness.

In Fig. 1 the propagation constants for the forward and backward propagation are plotted vs. the thickness of the guiding film. The separation between curves signifies the nonreciprocity of the waveguide structure. The difference of $\Delta \beta=\beta^{+}-\beta^{-}$decreases with increasing the film thickness. For nonreciprocal device applications it is important to achieve a large difference $\Delta \beta$. It is possible to increase $\Delta \beta$ by decreasing the refractive index step between the waveguiding film and the substrate.

In magnetooptic-nonlinear waveguide the nonreciprocal characteristics are affected by the intensity of the guided wave. A calculated difference between the 


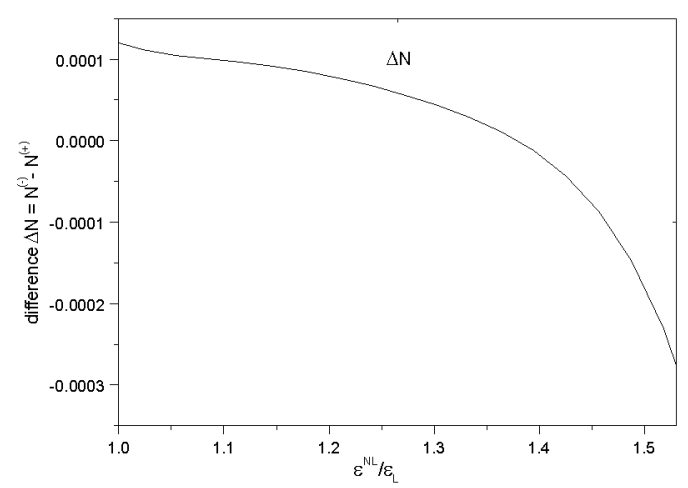

Fig. 2. Difference between the effective indexes $N^{( \pm)}=\beta^{( \pm)} / k_{0}$ for the forward and backward propagation of $\mathrm{TM}_{0}$ mode versus the nonlinear permittivity $\varepsilon^{\mathrm{NL}} / \varepsilon_{\mathrm{L}}$ at the interface film self-focusing nonlinear cover.

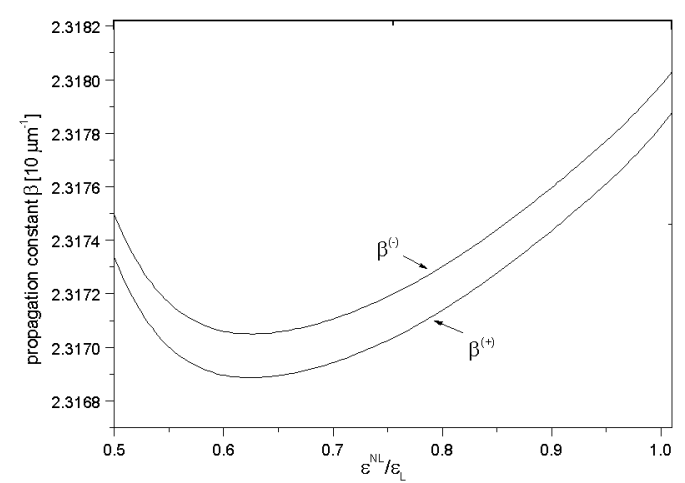

Fig. 3. Propagation constants for the forward and backward propagation of $\mathrm{TM}_{0}$ mode versus the nonlinear permittivity $\varepsilon^{\mathrm{NL}} / \varepsilon_{\mathrm{L}}$ at the interface film self-defocusing nonlinear cover.

effective indexes $\Delta N=N^{-}-N^{+}$for the backward and forward propagation of $\mathrm{TM}_{0}$ mode versus the boundary value of the nonlinear permittivity $\eta=\varepsilon^{\mathrm{NL}} / \varepsilon_{\mathrm{L}}$ in self-focusing $(\alpha>0)$ cladding is demonstrated in Fig. 2. We note that when $\eta$ reaches a value of 1.38 then $\Delta N$ changes the sign. It means that at a total power flow associated with this value of $\eta$ the nonreciprocal phase shift disappears [16]. The propagation constants for the forward and reverse travelling $\mathrm{TM}_{0}$ wave vs. the boundary value of the nonlinear permittivity $\eta=\varepsilon^{\mathrm{NL}} / \varepsilon_{\mathrm{L}}$ in a self-defocusing cover are presented in Fig. 3. The maximum difference $\beta^{(-)}-\beta^{(+)}$occurs near the region where both the propagation constants become a minimum. Figure 4 shows the variation of the normalized cut-off thickness of the forward and backward propagation with $\eta$ for the case of self-defocusing cladding. The cut-off thicknesses $w^{(+)}$and $w^{(-)}$increase as $\eta$ decreases from the linear limit $\eta=1$. They then go through a maximum at $\eta=0.63$ and, subsequently, decay as $\eta$ tends to the threshold value. The variation of cut-off thicknesses $w^{(+)}$and $w^{(-)}$for both the 


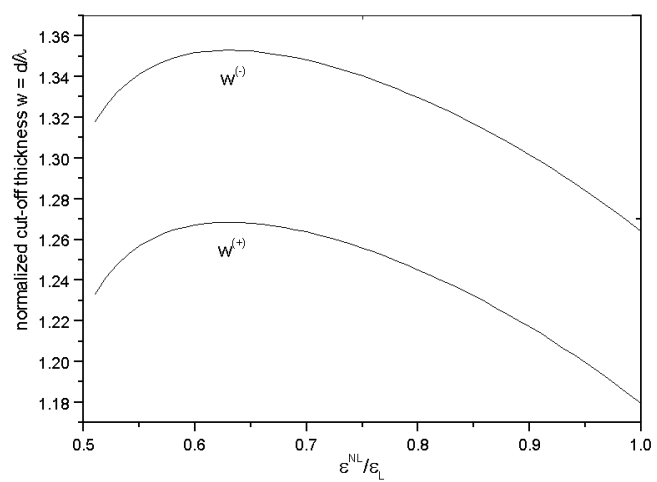

Fig. 4. Normalized cut-off thicknesses for the forward $\left(w^{(+)}\right)$and backward $\left(w^{(-)}\right)$ propagation of $\mathrm{TM}_{0}$ mode versus the nonlinear permittivity $\varepsilon^{\mathrm{NL}} / \varepsilon_{\mathrm{L}}$ at the interface film self-defocusing nonlinear cover.

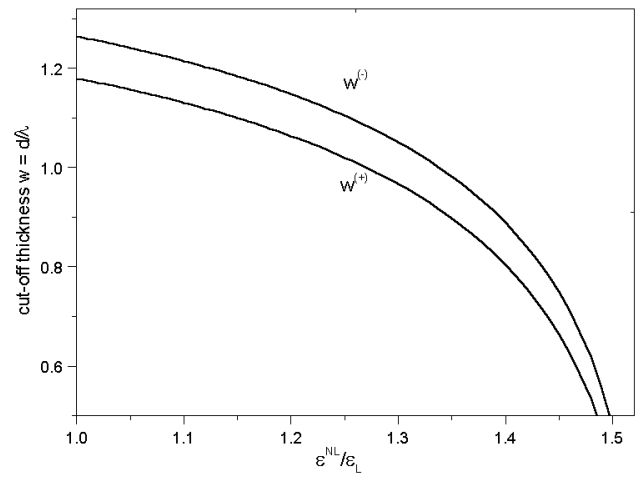

Fig. 5. Normalized cut-off thicknesses for the forward $\left(w^{(+)}\right)$and backward $\left(w^{(-)}\right)$ propagation of $\mathrm{TM}_{0}$ mode versus the nonlinear permittivity $\varepsilon^{\mathrm{NL}} / \varepsilon_{\mathrm{L}}$ at the interface film self-focusing nonlinear cover.

forward and the backward direction as a function of the boundary value $\eta$ in the focusing cladding is shown in Fig. 5. The values $w^{(+)}$and $w^{(-)}$decrease with an increase of local intensity (via the boundary value of $\eta$ ). Note that the difference $\Delta w=w^{(+)}-w^{(-)}$between the cut-off thicknesses for the forward and backward propagation does not depend on the boundary values of the power density.

\section{Conclusion}

We have presented a new configuration of the planar waveguide composed of a linear thin film sandwiched between the magneooptic substrate and Kerr-type nonlinear cladding. The theoretical and numerical analysis of the nonreciprocal behaviour of the TM modes propagating in the opposite directions has been carried out. With the help of the conservation energy law for TM modes in the nonlinear 
waveguide the dispersion relation without a knowledge of the nonlinear field profile has been derived. The nonreciprocal phase shift of the counterpropagating TM waves in such structure is sensitive to the power flow along the guide. The cut-off thicknesses for the forward and backward propagation are sensitive to the boundary value of the local intensity and consequently depend on the total power flow. If the light power reaches the magnitude for which the thickness of the waveguide becomes the cut-off value, say for the forward propagation, then only the reverse travelling is possible. Thus the structure acts as an intensity controlled isolator for TM waves. The proposed magnetooptic-nonlinear waveguiding structure can be applicable to the production of a new type of integrated devices utilizing both magnetooptic and nonlinear effects.

\section{References}

[1] L.A. Zenteno, Opt. Lett. 12, 657 (1987).

[2] K. Ando, Appl. Opt. 30, 1080 (1991).

[3] K. Matsubara, H. Yajima, J. Lightwave Technol. 9, 1061 (1991).

[4] J. Warner, IEEE Trans. Microw. Theory Tech. MTT-21, 769 (1973).

[5] Chin-Lin, A. Kumarswami, Appl. Opt. 25, 3664 (1986).

[6] H. Hemme, H. Dotsch, P. Hertel, Appl. Opt. 29, 2741 (1990).

[7] M. Shamonin, M. Lohmeyer, P. Hertel, H. Dotsch, Opt. Commun. 131, 37 (1996).

[8] G. Assanto, J. Mod. Opt. 37, 855 (1990).

[9] D. Mihalache, M. Bertolotti, C. Sibilia, in: Progress in Optics, Ed. E. Wolf, Vol. XXVII, North-Holland, Amsterdam 1989, p. 229.

[10] N. Akhemediev, in: Modern Problems in Condensed Matter Sciences, Vol. XXIX, Eds. H.E. Ponath, G.I. Stegeman, North-Holland, Amsterdam 1991, p. 289.

[11] A.D. Boardman, P. Egan, F. Lederer, U. Langbein, D. Mihalache, in: Nonlinear Surface Electromagnetic Phenomena, Eds. H.E. Ponath, G.I. Stegeman, North-Holland, Amsterdam 1991, p. 73.

[12] J. Jasiński, K. Gniadek, Opt. Quantum Electron. 36, 865 (1994).

[13] K. Gniadek, M. Rusek, in: Int. Conf. on Quantum Electronics Technical Digest Series, Vol. 9, Conference Edition, Vienna 1992, p. 426.

[14] K. Gniadek, M. Rusek, in: Technical Digest on Nonlinear Wave Phenomena, Vol. 15, Optical Society of America, Washington 1991, p. 100.

[15] K. Gniadek, Opt. Appl. 26, 381 (1996).

[16] K. Gniadek, Opt. Appl. 24, 281 (1994). 